\title{
Atmospheric Phase Characteristics of the ALMA Long Baseline
}

Satoki Matsushita ${ }^{a}$, Yoshiharu Asaki ${ }^{\mathrm{b}, \mathrm{c}}$, Edward B. Fomalont ${ }^{\mathrm{b}, \mathrm{d}}$, Denis Barkats ${ }^{\mathrm{e}}$, Stuartt A. Corder $^{\mathrm{b}, \mathrm{d}}$, Richard E. Hills ${ }^{f}$, Ryohei Kawabe ${ }^{\mathrm{g}, \mathrm{h}, \mathrm{i}}$, Luke T. Maud ${ }^{\mathrm{j}}$, Koh-Ichiro Morita ${ }^{\mathrm{b}, \mathrm{c}}$, Bojan Nikolic ${ }^{\text {, Remo P. J. Tilanus }}{ }^{\mathrm{j}}$, and Catherine Vlahakis ${ }^{\mathrm{b}, \mathrm{d}}$

a Academia Sinica Institute of Astronomy and Astrophysics, P.O. Box 23-141, Taipei 10617, Taiwan, R.O.C.

b Joint ALMA Observatory, Alonso de Córdova 3107, Vitacura 763 0355, Santiago, Chile 'National Astronomical Observatory of Japan, Chile Observatory, Alonso de Cordova 3107 , Vitacura 763 0355, Santiago, Chile

dNational Radio Astronomy Observatory, 520 Edgemont Rd, Charlottesville, VA 22903, USA 'Harvard-Smithsonian Center for Astrophysics, 60 Garden St., MS-78, Cambridge, MA 02138, USA

${ }^{\mathrm{f}}$ Astrophysics Group, Cavendish Laboratory, University of Cambridge, JJ Thomson Avenue, Cambridge CB3 0HE, UK

gNational Astronomical Observatory of Japan, 2-21-1 Osawa, Mitaka, Tokyo 181-8588, Japan

${ }^{\mathrm{h}}$ Department of Astronomy, School of Science, University of Tokyo, Bunkyo, Tokyo 113-0033, Japan

iSOKENDAI (The Graduate University for Advanced Studies), 2-21-1 Osawa, Mitaka, Tokyo 181-8588, Japan

jLeiden Observatory, Leiden University, P.O. Box 9513, 2300 RA Leiden, The Netherlands

\begin{abstract}
Atacama Large Millimeter/submillimeter Array (ALMA) is the world's largest millimeter / submillimeter (mm / submm) interferometer. Along with science observations, ALMA has performed several long baseline campaigns in the last 6 years to characterize and optimize its long baseline capabilities. To achieve full long baseline capability of ALMA, it is important to understand the characteristics of atmospheric phase fluctuation at long baselines, since it is believed to be the main cause of $\mathrm{mm} / \mathrm{submm}$ image degradation. For the first time, we present detailed properties of atmospheric phase fluctuation at $\mathrm{mm} / \mathrm{submm}$ wavelength from baselines up to 15 $\mathrm{km}$ in length. Atmospheric phase fluctuation increases as a function of baseline length with a power-law slope close to 0.6 , and many of the data display a shallower slope $(0.2-0.3)$ at baseline length greater than about 1 $\mathrm{km}$. Some of the data, on the other hand, show a single slope up to the maximum baseline length of around 15 $\mathrm{km}$. The phase correction method based on water vapor radiometers (WVRs) works well, especially for cases with precipitable water vapor (PWV) greater than $1 \mathrm{~mm}$, typically yielding a $50 \%$ decrease or more in the degree of phase fluctuation. However, significant amount of atmospheric phase fluctuation still remains after the WVR phase correction; about 200 micron in rms excess path length (rms phase fluctuation in unit of length) even at PWV less than $1 \mathrm{~mm}$. This result suggests the existence of other non-water-vapor sources of phase fluctuation, and emphasizes the need for additional phase correction methods, such as band-to-band and/or fast switching.
\end{abstract}

Keywords: ALMA, long baseline, atmospheric phase fluctuation, phase correction

Further author information: (Send correspondence to S.M.)

S.M.: E-mail: satoki@asiaa.sinica.edu.tw, Telephone: +886223665475

Ground-based and Airborne Telescopes VI, edited by Helen J. Hall, Roberto Gilmozzi, Heather K. Marshall, Proc. of SPIE Vol. 9906, 99064X · C 2016 SPIE · CCC code: 0277-786X/16/\$18 · doi: 10.1117/12.2231846 


\section{INTRODUCTION}

The Atacama Large Millimeter/submillimeter Array (ALMA) ${ }^{1}$ is the world's largest millimeter/submillimeter $(\mathrm{mm} / \mathrm{submm})$ interferometer. One of the most important technical developments for ALMA full operation was making the longest $(16 \mathrm{~km})$ baseline observations possible, which was achieved for the first time at the end of $2014 .^{2}$

Prior to that, the long baseline observations for $\mathrm{mm} / \mathrm{submm}$ linked arrays had been much shorter, $\sim 2 \mathrm{~km}$ at $230 \mathrm{GHz}$ with the Berkeley-Illinois-Maryland Association (BIMA) or the Combined Array for Research in Millimeter-wave Astronomy (CARMA), and several hundred meters at $345 \mathrm{GHz}$ or higher frequencies with the Submillimeter Array (SMA) or the NOrthern Extended Millimeter Array (NOEMA). The longest baseline length currently possible with ALMA $(\sim 16 \mathrm{~km})$, together with incredible numbers of baselines (1225 baselines wtih 50 antennas), is therefore a revolutionary improvement for $\mathrm{mm} / \mathrm{submm}$ linked arrays. This baseline length with good $u v$ coverage provides milli-arcsecond resolution $\mathrm{mm} / \mathrm{submm}$ data with a linked array, namely the data with high sensitivity and high fidelity that cannot be obtained with the other arrays. Indeed, in the ALMA long baseline campaign in 2014, science verification images revealed extraordinally detailed features of an asteroid, ${ }^{3}$ a protoplanetary disk system, ${ }^{4}$ and a gravitationally lensed system. ${ }^{5}$ In order to continue to improve the high quality of the data, it is important to test and characterize the atmospheric phase fluctuation at these long baselines.

Characterization of the atmospheric phase fluctuation so far was mainly done at a low frequency range; Very Large Array (VLA) was often used to characterize the atmospheric phase fluctuation at centimeter-wave (cmwave) with the baseline length up to a few tens of $\mathrm{km} .^{6,7}$ For higher frequency range, studies with $80-230$ $\mathrm{GHz}(1-3 \mathrm{~mm})$ are available with baseline length up to only about $1 \mathrm{~km} .{ }^{8-10}$ These past $\mathrm{cm}-/ \mathrm{mm}$-wave studies displayed similar results as far as the atmospheric phase fluctuation is caused by water vapor in the atmosphere. The longest baseline length of ALMA is therefore very important also for the study and characterization of the atmospheric phase fluctuation.

Since 2010, as a part of ALMA Commissioning and Science Verification (CSV), and most recently, as part of the Extension and Optimization of Capabilities (EOC), ${ }^{2}$ we have conducted several ALMA long baesline campaigns, starting from the longest baseline length of $600 \mathrm{~m}$ in 2010 and 2011, $2 \mathrm{~km}$ in 2012, $3 \mathrm{~km}$ in 2013 , and finally $10-15 \mathrm{~km}$ in 2014 . In the early phase (shorter baseline length) of the long baseline campaigns, we mainly conducted basic tests, such as characterizing the phase fluctuation and checking the effectiveness of the WVR phase correction, ${ }^{11,12}$ and in the later phase, we mainly concentrated on the evaluation of the phase correction methods. ${ }^{13,14}$ Overview of the latest $10-15 \mathrm{~km}$ baseline test has been reported in 2.

In this paper, we present the detailed characterization of phase fluctuation, improvement of phase fluctuation after the water vapor radiometer (WVR) phase correction method, ${ }^{15,16}$ and coherence time calculation using the data obtained in the past long baseline campaigns.

\section{OBSERVATIONS AND DATA REDUCTION}

All the data in this paper were taken with observations of a strong point source (i.e., radio-loud quasars) for tens of minutes (usually $10-40$ minutes, depending on the longest baseline length) with $\sim 1 \mathrm{~s}$ integration time per data point. Hereafter we refer to these observations as "single source stares." For each measurement, ten or more antennas with various baseline lengths have been used. We only used the $12 \mathrm{~m}$ diameter antenna data and did not use the data taken with the $7 \mathrm{~m}$ diameter antennas in order to make the thermal noise contribution uniform over the data as much as possible.

The single source stares up to 40 minutes were taken for the purpose of the statistical phase analysis beyond the wind crossing time of the longest baseline. Assuming (1) a longest baseline length of $10 \mathrm{~km},(2)$ a wind speed along the baseline of $10 \mathrm{~m} \mathrm{~s}^{-1}$, and (3) phase screen does not change with time (frozen phase screen), ${ }^{17,18}$ then the crossing time can be calculated as $1000 \mathrm{~s}(\sim 17$ minutes $)$. To have statistically significant data for the longest baseline data, about twice the measurement time is needed (see 19 for the detailed discussion). We used Bands 3 (100 GHz band), 6 (200 GHz band), and 7 (300 GHz band) for all the campaigns, except for Band 8 (400 $\mathrm{GHz}$ band), which has only been used in the 2014 campaign. 
The data have been reduced on the Common Astronomy Software Applications (CASA) package, ${ }^{20}$ using a standard ALMA calibration prescription. The WVR phase correction has been applied using the program wvrgcal $^{21}$ installed inside CASA. Note that the precipitable water vapor (PWV) has also been calculated by wvrgcal, and we use this PWV as the water vapor content in front of the array of each data set.

Linear phase drift has then been taken out from each data, which is mostly caused by the baseline determination error. This error is generally small enough that it only causes the linear phase drift over of order tens of minutes. After this, we binned the data points for $10 \mathrm{~s}$ to take out the effect of the thermal noise in the recieving system, which is suggested in the "ALMA System Technical Requirements for $12 \mathrm{~m}$ array". ${ }^{22}$ It is known that the phase fluctuation is dominated by the atmospheric effect even at very short baseline length of about $15 \mathrm{~m},{ }^{11}$ and the smallest phase fluctuation observed before the WVR phase correction is about $6 \mu \mathrm{m}$, which is much smaller than what we observed here. The thermal noise is smaller than this value, and indeed much smaller than the value quoted in 22 , so we disregarded the effect of thermal noise in our data. At the $10 \mathrm{~s}$ data binning process, if the data points are less than $70 \%$ of the number of the data points should be (i.e., in case of 1 second integration time per data point, then less than 7 data points), then this binned data have been flagged out. This $10 \mathrm{~s}$ binned data have been used for further calculations.

We then calculated the root mean square (rms) phase fluctuation for each baseline. For the unit of phase, we express in path length:

$$
\Phi=\frac{\theta}{360^{\circ}} \times \frac{c}{\nu},
$$

where $\Phi$ and $\theta$ are phase in path length and in degrees, respectively, $c$ is the speed of light, and $\nu$ is the observation frequency. This is to directly compare the results from various frequencies in the same unit without any frequency dependence, and is often referred to as excess path length.

\section{RESULTS AND DISCUSSION}

\subsection{Improvement Factor of the WVR Phase Correction}

The improvement factor of the WVR phase correction method has been calculated by dividing the rms excess path length without the WVR phase correction by that with the correction for each baseline. Higher values mean that the WVR phase correction works better (improved more) than lower value (less improvement) cases. Improvement factor less than unity means that the WVR phase correction made the phase fluctuation worse than the original data. The calculated values are then averaged over all baselines, and plotted as a function of PWV (Fig. 1a). We also made another plot with the data separated into two, one with baseline lengths shorter than $1 \mathrm{~km}$ (Fig. 1b blue symbols) and the other with baseline length $>1 \mathrm{~km}$ (Fig. 1b red symbols). For the former plot, we included 2012 - 2014 data $(2 \mathrm{~km}, 3 \mathrm{~km}$, and $10-15 \mathrm{~km}$ baseline campaign), but for the latter, we only included 2014 data. This is because the 2012 - 2013 data have very limited numbers of long baseline antennas (for the 2012 campaign, only 1 antenna was on a long baseline, and for the 2013 campaign, only 4 antennas), and therefore the results can be highly biased on the antenna-based characteristics, such as the WVR instrumental characteristics of each antenna, rather than the statistical nature of the WVR phase correction method.

For the plot averaged over all the baselines (Fig. 1a), it is obvious that the improvement factor is high when PWV is larger than $1 \mathrm{~mm}$ : The overall improvement factor is $2.1 \pm 0.7$, but in case of PWV $<1 \mathrm{~mm}$, the ratio is $1.7 \pm 0.4$, and in case of $\mathrm{PWV}>1 \mathrm{~mm}$, it increases to $2.4 \pm 0.7$. This means that the improvement factor tends to be low when PWV is lower than $1 \mathrm{~mm}$. On the other hand, when PWV is larger than $1 \mathrm{~mm}$, the improvement factor tends to be higher, namely the WVR phase correction works better. These results can be explained as follows; when PWV is low, the difference of the amount of water vapor along the line of sight of two antennas is not large compared to other constituent(s) (see Sect. 3.2.5 for discussions about the other constituents).

Fig. 1(b) shows that the improvement factor for the baseline length shorter than $1 \mathrm{~km}$ is $2.3 \pm 0.9$, and that for the baseline length longer than $1 \mathrm{~km}$ is $2.0 \pm 0.7$. The longer baseline data tend to have lower improvement factor than the shorter ones, although the difference is statistically not significant.

In both plots, different frequency bands are plotted with different symbols, but we find no significant difference in the improvement factor as a function of frequency bands. 

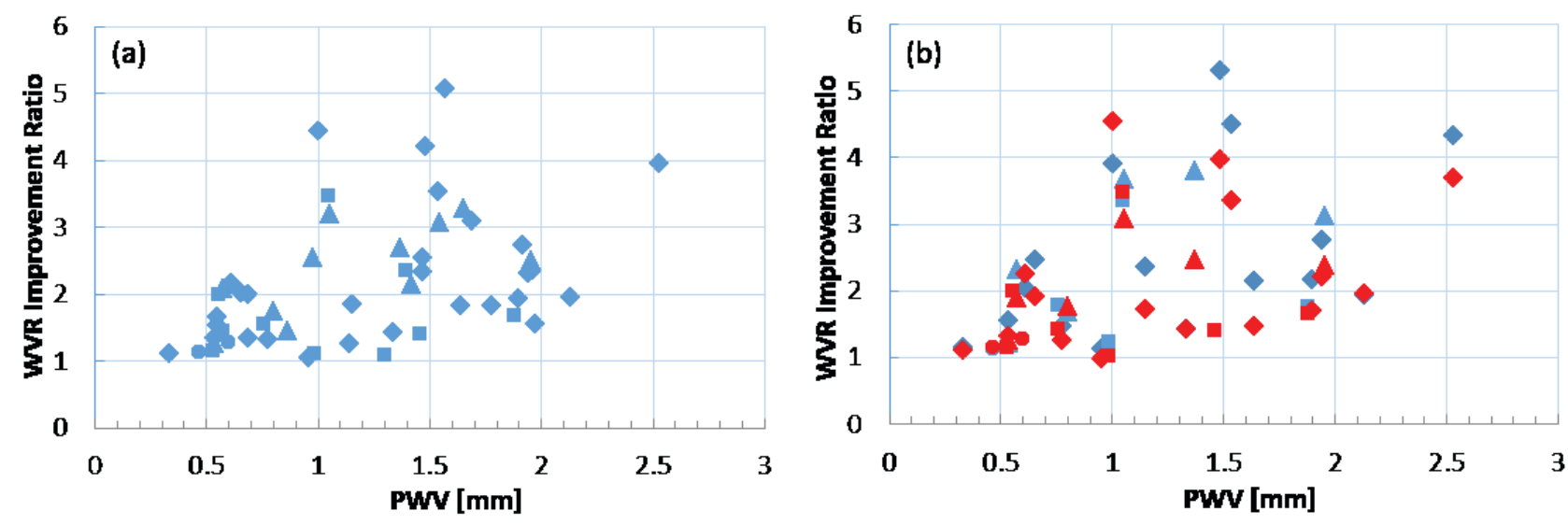

Figure 1. Improvement factor of the WVR phase correction (rms excess path length without the WVR phase correction divided by that with the correction) as a function of PWV. Symbols are differentiated with the frequency bands; diamond, triangle, square, and circle symbols indicate the data taken with Bands 3, 6, 7, and 8, respectively. (a) Each data point is averaged over all baselines. This plot uses all the 2012 - 2014 data. (b) Data points have been separated for the one averaged over the baseline length shorter (blue) or longer (red) than $1 \mathrm{~km}$. This plot only uses the 2014 data, since only the 2014 data have statistically significant data points at baselines longer than $1 \mathrm{~km}$.

\subsection{Spatial Structure Function of Phase Fluctuation}

In this subsection, we first define the spatial structure function (SSF) of phase (Sect. 3.2.1), and derive the SSF slopes for all the past long baseline data (Sect. 3.2.2). This statistical result is compared with the past studies (Sect. 3.2.2). We then estimate the degree of phase fluctuation for the baseline length at $10 \mathrm{~km}$, and derive a weak correlation with PWV (Sect. 3.2.3). Empirical relations between the phase fluctuation and the baseline length are also derived (Sect. 3.2.4). Large amount of phase fluctuation has not been taken out after the WVR phase correction, suggesting other components than water vapor that cause the phase fluctuation exist in the atmosphere; we discuss the possibilities for liquid water, ice, and dry components (Sect. 3.2.5), and also any instrumental causes.

\subsubsection{Definition of Spatial Structure Function}

SSF of phase is defined as

$$
D_{\theta}(d)=\left\langle\{\theta(x)-\theta(x-d)\}^{2}>,\right.
$$

where $\theta(x)$ and $\theta(x-d)$ are phases at positions $x$ and $x-d$, and the angle brackets mean an ensemble average. ${ }^{23,24}$ Since $\theta(x)-\theta(x-d)$ is an interferometer phase with baseline length $d, \sqrt{D_{\theta}(d)}$ is approximated to the rms phase of a baseline over the entire observation time. SSF plots in this paper have therefore been made with calculating the rms phase for each baseline using of tens of minutes of observation time, and plotted as a function of baseline length.

Slopes for SSFs are well studied theoretically, and in case of 3-dimensional (3-D) Kolmogolov turbulence of the Earth's atmosphere, it is expected to have a slope of 0.83 ; for the 2-D turbulence case, the slope is expected to be 0.33 . In case of no correlation in the atmospheric turbulence between two antennas, the slope is expected to be zero.

Fig. 2 shows two example plots of SSFs we obtained using the ALMA data. In these plots, both the WVR phase corrected (red) and uncorrected (blue) SSFs are displayed. As mentioned above, phase is converted into the unit of path length. Fig. 2(a) displays a typical SSF plot; there is a clear turn-over in the SSF at baseline lengths of around $1 \mathrm{~km}$ in both plots with and without the WVR phase correction. Shorter baselines before the turn-over show a steeper slope, while longer baselines show a shallow or almost flat slope. The turn-over is often observed around a baseline length of several hundred meters to $\sim 1 \mathrm{~km}$, but those data only display one turn-over under the current data reduction method. Fig. 2(b), on the other hand, exhibits no clear turn-over; 

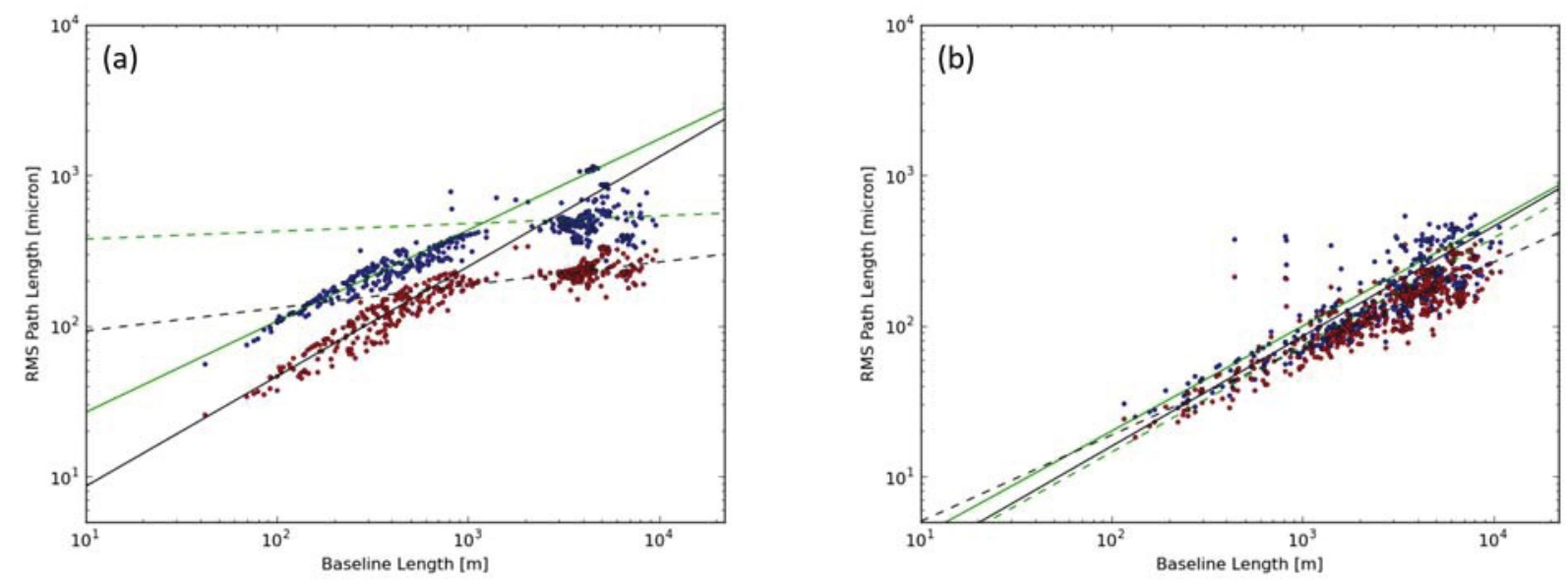

Figure 2. Two representative plots of the spatial structure function (SSF) with (red) and without (blue) WVR phase correction. Solid and dashed lines are the fitting results of slopes using baselines shorter than $500 \mathrm{~m}$ and longer than 1 $\mathrm{km}$, respectively. (a) Example of SSF with a clear turn-over. (b) Example of SSF without any clear turn-over. Note that for the SSF without any clear turn-over, there is almost no improvement with the WVR phase correction (i.e., red and blue dots are almost overlapping each other).

phase fluctuation increases constantly even at long baselines. Such a constant slope SSF is not common, but is sometimes observed in the data (see Sect. 3.2.2 for statistics).

The baseline length of the turn-over roughly corresponds to the scale height of the turbulent constituent in the atmosphere. Our results suggest that the scale height of the turbulent constituent at the ALMA site is about $1 \mathrm{~km}$ in most cases, which is a typical value for water vapor in the atmoshpere. The main turbulent constituent at the ALMA site is therefore highly possible water vapor, which is consistent with the improvement of phase fluctuation by the WVR phase correction in most cases (Sect. 3.1). On the other hand, no turn-over means that the scale height of the turbulent constituent is higher than $10 \mathrm{~km}$. This scale height is much higher than that of water vapor, suggesting that the turbulent constituent for the SSF with no turn-over is caused by other constituent (see Sect. 3.2.5 for further discussion).

\subsubsection{Slopes for the Spatial Structure Functions}

We first fitted the slopes for the data points with baseline lengths shorter than $500 \mathrm{~m}$ and longer than $1 \mathrm{~km}$ for each SSF plot, namely shorter and longer than turn-overs in the plots. The fitting function is expressed as

$$
\log _{10}(\Delta L)=a \times \log _{10}(d)+b,
$$

where $\Delta L$ is the rms excess path length in micron, $d$ is the baseline length in meter, and $a$ and $b$ are the slope, namely the structure exponent, and the structure constant, respectively. Examples of the fitting are shown in Fig. 2, and the fitted slopes as a function of PWV are plotted in Fig. 3; (a) is for the shorter baseline slopes, and (b) for the longer baseline slopes.

In Fig. 3(a), there is no significant trend in either as a function of PWV, frequency, or the effect of the WVR phase correction. The fitted slopes before and after the WVR phase correction with averaging all the

Table 1. Fitted slopes of the spatial structure functions (SSFs).

\begin{tabular}{|c|c|c|c|c|c|c|}
\hline & \multicolumn{3}{|c|}{ Without WVR } & \multicolumn{3}{c|}{ With WVR } \\
\hline Baseline Length & All & PWV $<1 \mathrm{~mm}$ & PWV $>1 \mathrm{~mm}$ & All & PWV $<1 \mathrm{~mm}$ & PWV $>1 \mathrm{~mm}$ \\
\hline$<500 \mathrm{~m}$ & $0.65 \pm 0.06$ & $0.65 \pm 0.06$ & $0.66 \pm 0.06$ & $0.62 \pm 0.09$ & $0.64 \pm 0.08$ & $0.60 \pm 0.09$ \\
\hline$>1 \mathrm{~km}$ & $0.22 \pm 0.15$ & $0.27 \pm 0.20$ & $0.17 \pm 0.07$ & $0.29 \pm 0.13$ & $0.31 \pm 0.15$ & $0.26 \pm 0.11$ \\
\hline
\end{tabular}



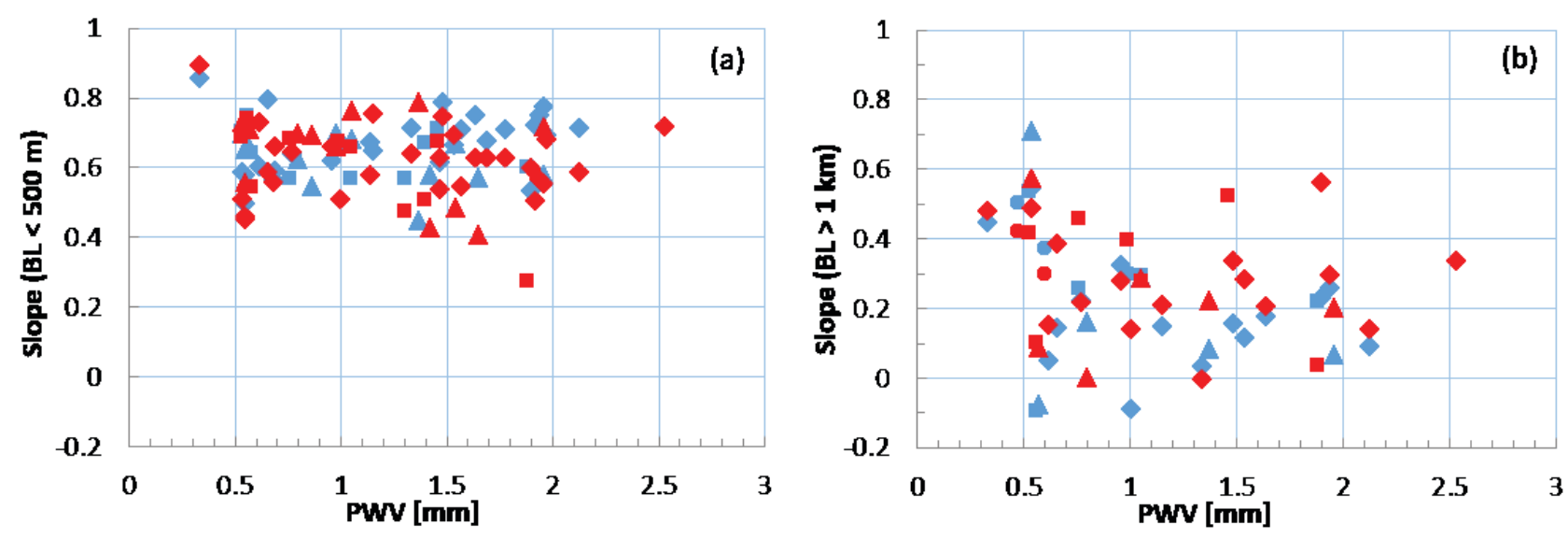

Figure 3. Fitted slopes of the spatial structure functions (SSFs) as a function of PWV. Slopes for the data points in each SSF with the baseline lengths (a) shorter than $500 \mathrm{~m}$, and (b) longer than $1 \mathrm{~km}$. Symbols are differentiated with the frequency bands; diamond, triangle, square, and circle symbols indicate the data taken with Bands 3, 6, 7, and 8, respectively. Blue symbols are the data before the WVR phase correction, and red after. Note that (a) includes the 2012 - 2014 data, but (b) only includes the 2014 data, for the reason same as in Sect. 3.1.

data, and that with PWV lower and higher than $1 \mathrm{~mm}$ are shown in the top row of Table 1 . All those values are around 0.6 , indicating that the WVR phase correction or the amount of water vapor in the atmosphere does not change the shorter baseline slopes of SSFs. The average slope without the WVR phase correction of $0.65 \pm 0.06$ is consistent with the $50 \%$ quartile slope for the 3-year (1996 July - 1999 March) statistical data using the site testing $11.2 \mathrm{GHz}$ radio seeing monitor of $0.63 .{ }^{25}$ This indicates that the data we took are typical phase fluctuation characteristics at the ALMA site.

Fig. 3(b) clearly shows that the slopes at longer baselines (baseline length $>1 \mathrm{~km}$ ) are shallower than the shorter baselines (baseline length $<500 \mathrm{~m}$; Fig. 3a) in most cases. Similar as above, the fitted slopes are shown in the bottom row of Table 1. The average slopes are $0.22 \pm 0.15$ and $0.29 \pm 0.13$ for the data before and after the WVR phase correction, respectively. These values are significantly smaller than those for the baseline length shorter than $500 \mathrm{~m}$, indicating that most of the data have a turn-over at the baseline length between $500 \mathrm{~m}$ and $1 \mathrm{~km}$.

For almost all the cases, the slopes for shorter baselines are in the middle of theoretical 3-D and 2-D Kolmogorov turbulence, which are 0.83 and 0.33 , respectively. This may suggest the existence of undeveloped turbulence or multi-layer 2-D turbulence in the atmosphere. The slopes for longer baselines (slopes after the turn-over), on the other hand, exhibit the values between the 2-D Kolmogorov turbulence (slope $=0.33$ ) and no correlation between two antennas (slope $=0)$ in most cases.

Our result is very similar to the result from the statistical study of the SSF slope at the VLA site; the slope of 0.59 at short $(<1 \mathrm{~km})$ baselines, and 0.3 at longer baselines. ${ }^{6}$ On the other hand, later SSF study using VLA exhibits two clear turn-overs, one from the 3-D Kolmogorov turbulence (slope $=0.83$ ) to the 2-D one $($ slope $=0.33)$, and the other from the 2-D one to the no-correlation regime (slope $=0) .{ }^{7}$ SSFs with two turn-overs has never been obtained in the previous statistical study, suggesting that SSFs with two turn-overs are statistically rare, or the coverage of the array configuration was not wide enough to investigate the full range of the atmospheric turbulence.

For PWV $<1 \mathrm{~mm}$, the average of the slope at longer baselines is much smaller than that for the shorter baselines, but the scatter of the slope is large, and the highest values of the slope are almost the same as that of the shorter baselines. This means that there are some cases that does not have any turn-over, as an example SSF plot shown in Fig. 2(b). As mentioned in Sect. 3.1, at this low PWV range, the WVR phase correction does not work well, suggesting that the phase fluctuation is not due to water vapor but caused by other constituent, similar conclusion as above (Sect. 3.2.1). 


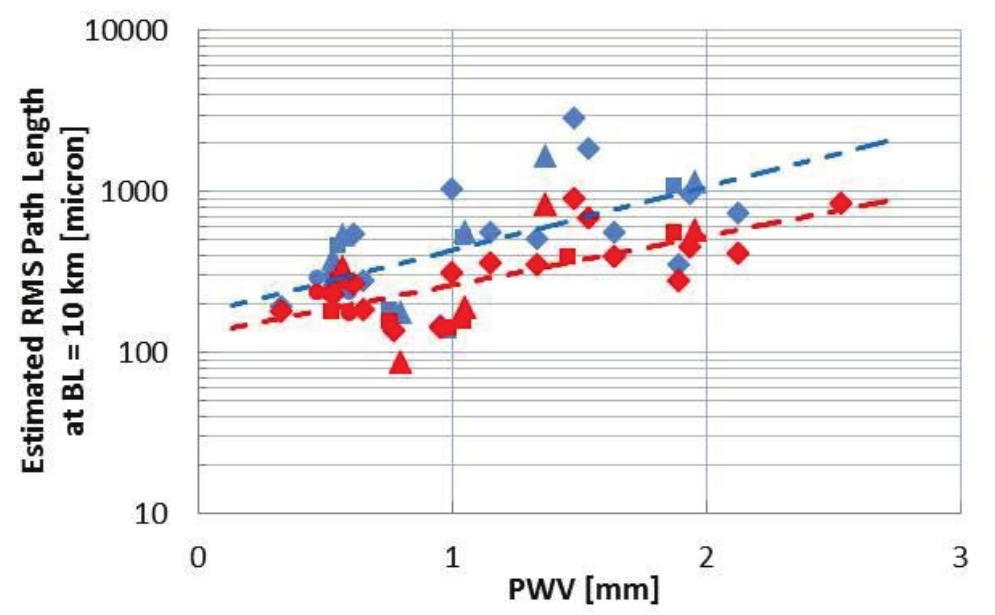

Figure 4. Estimated rms excess path length of each data set taken in 2014 at the baseline length of $10 \mathrm{~km}$ using the fitted results mentioned in Sect. 3.2.2, which roughly represents the rms excess path length even longer than $10 \mathrm{~km}$. Blue and red dashed lines are the fitting results for the estimated rms excess path length before and after the WVR phase correction, respectively. Symbols and their colors are the same as in Fig. 3.

For PWV > $1 \mathrm{~mm}$ and without the WVR phase correction, the slopes are always small (small values with small scatter), but after the WVR phase correction, both the slope and the scatter being large. This, together with the improvement factor of the WVR phase correction mentioned above (Sect. 3.1), indicates that many of the cases the slopes turn to be steeper after the WVR phase correction for the longer $(>1 \mathrm{~km})$ baselines. For some cases, the slopes turn to be similar as the one at the shorter $(<500 \mathrm{~m})$ baselines, namely SSF being similar to the one with no turn-over, suggesting that the WVR phase correction took out all the phase fluctuation caused by water vapor, and that caused by other consituent remains. This means that if we can completely remove the phase fluctuation due to water vapor in the atmosphere, a large-scale turbulent component that covers the longest baseline length reveals.

\subsubsection{Phase Fluctuation at $10 \mathrm{~km}$ Baseline}

To understand quantitatively how much phase fluctuation exists at long baselines, we calculate the rms excess path length for the baseline length at $10 \mathrm{~km}$. Although the averaged slope at baseline length longer than $1 \mathrm{~km}$ is around $0.2-0.3$ (see Sect. 3.2.2), the slope at baseline length longer than $10 \mathrm{~km}$ might be close to zero, namely no increase of the rms excess path length. ${ }^{7}$ Therefore it is expected that the rms excess path length at $10 \mathrm{~km}$ is roughly equal to the rms excess path length longer than $10 \mathrm{~km}$. Here, we estimate the rms excess path length at $10 \mathrm{~km}$ using the fitting results derived in the previous subsection. This information will be useful for high frequency observations, or future extended-ALMA, ${ }^{26}$ which is to extend the maximum baseline lengths longer than the current ALMA (>16 km), or Very Long Baseline Interferometry (VLBI) observations, which will have much longer baseline lengths $(>100 \mathrm{~km})$.

Using Eq. (3) to fit the data with baseline length longer than $1 \mathrm{~km}$, we estimated the rms excess path length at the baseline length of $10 \mathrm{~km}$ for each data set. Fig. 4 displays the estimated rms excess path length at the baseline length of $10 \mathrm{~km}$ as a function of PWV for both before (blue) and after (red) the WVR phase correction. As can be seen, both data show weak correlations with PWV (see the dashed lines for the fitting results); lower PWV conditions tend to have smaller rms excess path length, and opposite for higher PWV conditions. This is understandable since a larger amount of water vapor in the atmosphere causes larger phase fluctuation. On the other hand, the scatter is large, almost an order of magnitude at a given PWV ranges, which makes the correlation weak. This indicates that the phase fluctuation cannot only be the fucntion of the total water vapor content in the atmosphere.

For almost all the data, there is a significant rms excess path length after the WVR phase correction, with a mean value of $206 \mu \mathrm{m}$ even at PWV $<1 \mathrm{~mm}$. This result indicates that even after the WVR phase correciton 

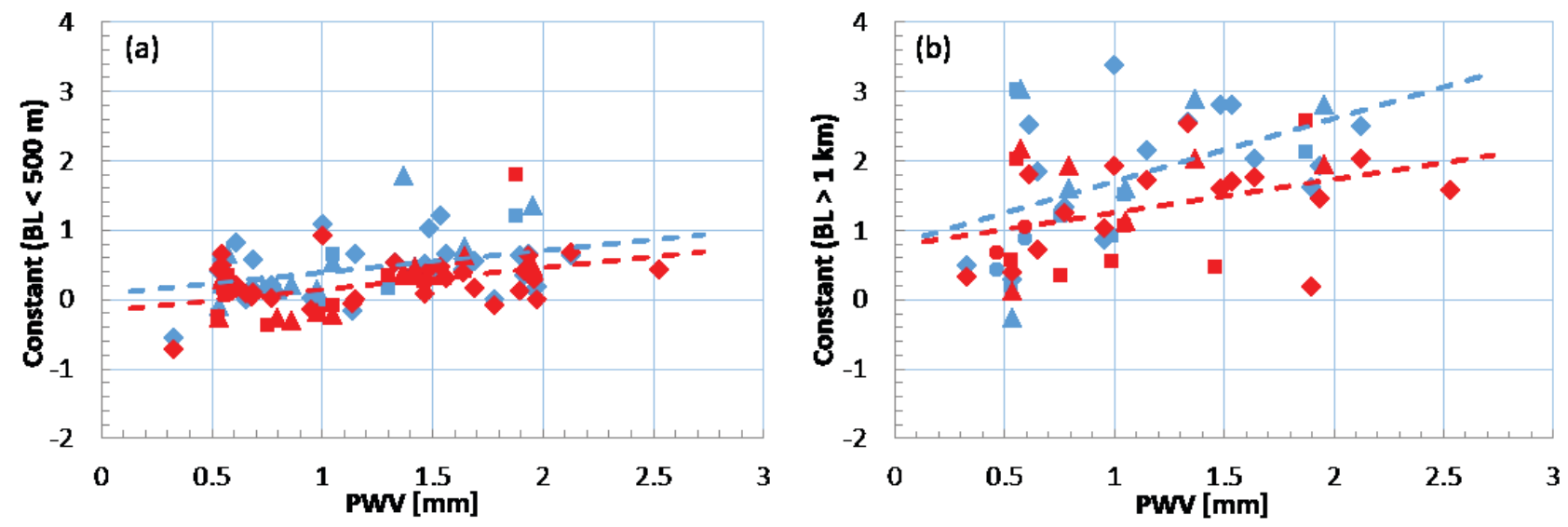

Figure 5. Fitted structure constants of SSFs as a function of PWV. Structure constants for the data points in each SSF with the baseline lengths (a) shorter than $500 \mathrm{~m}$, and (b) longer than $1 \mathrm{~km}$. Blue and red dashed lines are the fitting results for the data before and after the WVR phase correction, respectively. Symbols and their colors are the same as in Fig. 3.

under good weather conditions, peak-to-peak phase fluctuation reaches around $2 \pi$ or more at the high frequency bands (i.e., Bands $9 \& 10 ; 600-1000 \mathrm{GHz}$ or $300-500 \mu \mathrm{m}$ ). This phase fluctuation blurs the final synthesized image with the blurring size around twice the synthesized beam size or more, significantly affects the imaging quality, if we calibrate the phase at the same frequency. This result strongly suggests that calibration can be improved by calibrating at lower frequency and applying the solutions to higher frequency (i.e., band-to-band phase calibration), and/or using the fast switching phase correction methods. ${ }^{7,14,27}$

\subsubsection{Relation between Baseline Length and the RMS Excess Path Length}

Since the slope $a$ in Eq. (3) does not show any correlation with PWV (Fig. 3a), but the rms phase at $10 \mathrm{~km}$ baseline weakly does (Fig. 4), it suggests that the structure constant $b$ in Eq. (3) is expected to have weak correlation with PWV. Figs. 5(a) and (b) display the derived constants as a function of PWV for baseline lengths shorter than $500 \mathrm{~m}$ and longer than $1 \mathrm{~km}$, respectively. For both cases, there is a weak correlation between the structure constants and PWV for both before and after the WVR phase correction (Fig. 5), similar as the correlation between the rms phase at $10 \mathrm{~km}$ and PWV (Fig. 4).

For the shorter baselines, the correlation is displayed with the dashed lines in Fig. 5(a), and can be expressed as

$$
b_{\text {raw }}=0.3 \times \mathrm{PWV}+0.1
$$

and

$$
b_{w v r}=0.3 \times \mathrm{PWV}-0.2
$$

for the data before and after the WVR phase correction, respectively. For the longer baselines, the correlation in Fig. 5(b) can be expressed as

$$
b_{\text {raw }}=0.9 \times \mathrm{PWV}+0.8
$$

and

$$
b_{w v r}=0.5 \times \mathrm{PWV}+0.8
$$

for the data before and after the WVR phase correction, respectively.

Using these derived slopes and constants in Eq. (3), it is possible to formulate the empirical relationship between the rms excess path length and the baseline length at the ALMA site. For the baseline length shorter 
than $\sim 1 \mathrm{~km}$, where the slope is generally steep, since there is no dependence on PWV, it is possible to express the rms excess path length using Eqs. (3), (4), and (5), and the average of the slopes mentioned above as

$$
\log _{10}\left(\Delta L_{\text {short,raw }}\right)=0.65 \times \log _{10}\left(d_{<1 k m}\right)+0.3 \times \mathrm{PWV}+0.1
$$

for the data before the WVR phase correction, and

$$
\log _{10}\left(\Delta L_{\text {short }, w v r}\right)=0.65 \times \log _{10}\left(d_{<1 k m}\right)+0.3 \times \mathrm{PWV}-0.2
$$

for the data after the WVR phase correction.

For the baseline length longer than $\sim 1 \mathrm{~km}$, where the slope is generally shallow, equations can be formulated using the averaged values of the slope and Eqs. (3), (6), and (7) as

$$
\log _{10}\left(\Delta L_{\text {long,raw }}\right)=0.22 \times \log _{10}\left(d_{>1 \mathrm{~km}}\right)+0.9 \times \mathrm{PWV}+0.8
$$

for the data before the WVR phase correction, and

$$
\log _{10}\left(\Delta L_{\text {long,wvr }}\right)=0.29 \times \log _{10}\left(d_{>1 \mathrm{~km}}\right)+0.5 \times \mathrm{PWV}+0.8
$$

for the data after the WVR phase correction. With these empirical equations, it is possible to estimate the rms excess path length on any baseline length for the current ALMA (i.e., baseline length up to $15 \mathrm{~km}$ ). Note that the scatter is large so that this estimation is useful only to tell the tendency of the phase fluctuation.

\subsubsection{Why the WVR Phase Correction Cannot Take Out All the Phase Fluctuation?}

In most cases, the main difference between SSFs with and without the WVR phase correction is the absolute rms phase values, and the overall feature does not change significantly with the WVR phase correction and only weakly correlated with PWV. If the WVR phase correction takes out all the phase fluctuation, the resultant spatial structure function should exhibited an almost flat feature; since the WVR phase correction applies every 1 second to the data, if the wind blows along a baseline with its velocity of $10 \mathrm{~m} \mathrm{~s}^{-1}$ and assuming a frozen phase screen, then the phase at the baselines longer than $10 \mathrm{~m}$ should show random phase that corresponds to the thermal noise of the WVRs. We have never observed such SSF in the last 5 years of the long baseline campaigns (see also 11,12). The overall similarity between the SSFs with and without the WVR phase correction suggests that the origin of the phase fluctuation is not only due to the water vapor but also other turbulent constituents. Furthermore, SSFs without any turn-over also suggests the existence of other turbulent constituents with the scale height higher than the longest baseline length of $\sim 10 \mathrm{~km}$, as mentioned above (Sect. 3.2.1,3.2.2). Indeed, this higher scale height turbulent constituent has also been independently suggested by other measurements. ${ }^{28}$

The cause of this remaining phase fluctuation is still unclear: Liquid water (fog or clouds) in the atmosphere absorbs the amplitude of electromagnetic wave significantly ${ }^{29-31}$ at continuum level. This changes the line profile of the water vapor in the atmosphere, so that the WVR phase correction, which uses the liquid water-free line profile model, will no longer be appropriate. ${ }^{32,33}$ Therefore if liquid water dominates the atmosphere, then rms phase after the WVR phase correction does not improve or often makes it worse. Since we are looking for the cause of the remaining phase fluctuation after the WVR phase correction, liquid water cannot be the cause. Water ice in the atmosphere, which cannot be detected by WVRs, can induce similar phase fluctuations as water vapor, ${ }^{34,35}$ but while it could potentially be one of the causes, it is not clear whether it always exist in the atmosphere. A dry component (i.e., $\mathrm{N}_{2}$ and $\mathrm{O}_{2}$ ) in the atmosphere could also be the cause of density fluctuations, ${ }^{16}$ but it is still not confirmed observationally. But both water ice and dry component have a high scale height of around $10 \mathrm{~km}$ or more, so that the SSFs with no turn-over can be caused by these constituents.

Instrumentation problems can also be possible; since SSF is baseline-based, instruments that can cause baseline-based problems needed to be considered. Correlator is one of the baseline-base instruments, but it is very doubtful to produce larger phase fluctuation at longer baselines all the time. Line Length Corrector (LLC; round-trip phase corrector) is an instrument that can create phase noise as a function of baseline length; noise in this instrumental component causes antenna-base phase fluctuation, but since the relative effect is large for shorter baseline and small for longer baseline, it appears as baseline-based. However, the variation of LLC in each antenna is always monitored, and the timescale of the variation is much longer than the observed phase fluctuation. This suggests that LLC is highly unlikely to cause the phase fluctuation. 


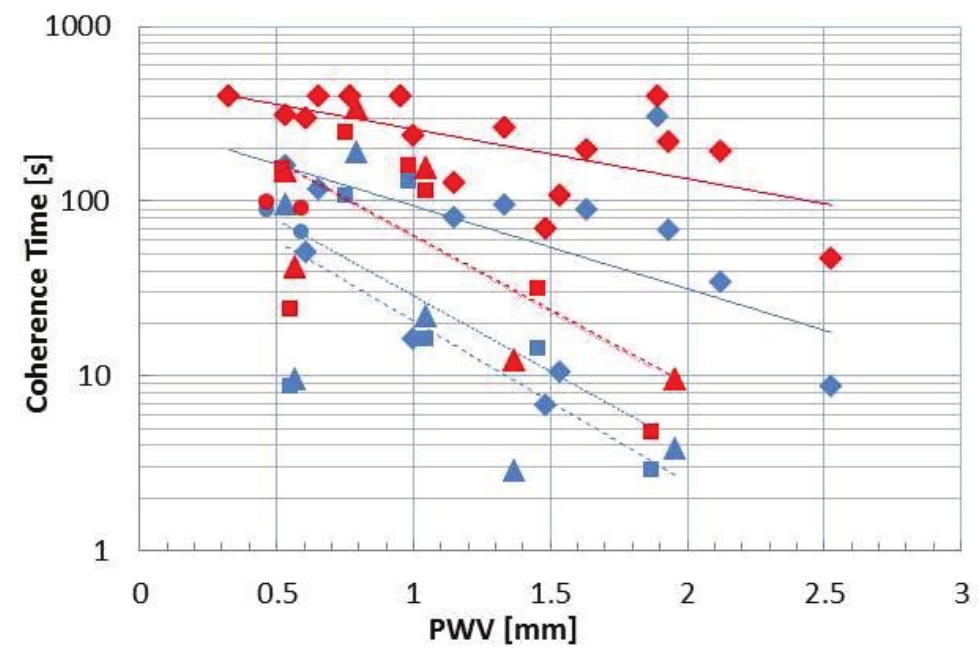

Figure 6. Coherence time to be the coherence of 0.9 (i.e., coherence loss of 10\%) as a function of PWV. Each data point in the plot is a median of the coherence time of each baseline with its length longer than $1 \mathrm{~km}$ in each data set. Solid, dashed, and dotted lines are the fitted lines for Bands 3, 6, and 7 data, respectively. Blue lines are for the data before the WVR phase correction, and red lines after. Symbols and their colors are the same as in Fig. 3.

\subsection{Coherence Time}

Temporal coherence function is defined as

$$
C(T)=\left|\frac{1}{T} \int_{0}^{T} \exp \{-i \theta(t)\} d t\right|,
$$

where $\theta(t)$ is phase at a time $t$ and $T$ is an arbitrary integreation time. ${ }^{24}$ This equation is namely vector averaging of phase in time domain. A coherence of unity means no loss in coherence (i.e., amplitude) due to the phase fluctuation, and that of zero means no coherence at all due to huge phase fluctuation. Coherence time is a maximum $T$ with which the coherence is not smaller than a certain critical value; since phase fluctuation increases as time passes from a certain time, coherence decreases.

Coherence time is highly related to an integration time for one data point for radio arrays; if one can torelate a certain coherence loss, one could define the integration time based on the coherence time. This is also true for the calibration time; for a typical interferometric observation, a phase calibrator will be observed at certain time intervals, and if one can torelate a certain coherence loss, one could define the calibration time based on the coherence time.

Furthermore, this is also highly related to the integration time for VLBI using ALMA (ALMA Phase-Up Project, and also for using the single dish telescopes around ALMA, namely APEX and ASTE); for detecting interferometric signals (i.e., fringes) with VLBI, it is important to have high signal-to-noise ratio (S/N) within one data point, since it is needed to search the signal in the ranges of delay and delay rate due to uncertainty in the antenna locations. On the other hand, since the atmosphere will affect the VLBI phase stability significantly, we should be careful in selecting the integration time for VLBI observations, especially in $\mathrm{mm} / \mathrm{submm}$ wave. The coherence time is a good suggestive integration time for the signal detection with VLBI.

Since the phase fluctuation depends on the baseline length, but the slope will be significantly shallow at baseline lengths longer than $1 \mathrm{~km}$ (see Sect. 3.2), we take a median value of coherence time derived from the data at the baseline lengths longer than $1 \mathrm{~km}$. Here, we calculate the coherence time that degrades the coherence to 0.9 (i.e., coherence loss of 10\%). The calculation results are shown in Fig. 6 as a function of PWV. Each calculation stops at the time range of $400 \mathrm{~s}$, since ALMA will not calibrate phase in such a long duration. So the data points located at $400 \mathrm{~s}$ means that these did not reach the coherence loss of $10 \%$ even after $400 \mathrm{~s}$. It 
is obvious that the data after the WVR phase correction have longer coherence time than that without. The average of the coherence time after the WVR phase correction is $184 \mathrm{~s}$, about twice longer than that without of $101 \mathrm{~s}$. This indicates that the WVR phase correction improves the coherence time of about twice or more (since our calculation stops at $400 \mathrm{~s}$, the coherence time is underestimated, especially for the WVR phase corrected data, which have more data that reached $400 \mathrm{~s}$ ). The WVR phase correction is therefore useful for extending integration or calibration time.

In terms of overall PWV dependency of coherence time, there is a weak trend for lower PWV to give rise to longer coherence time. This can be understood as more water vapor in the atmosphere, more phase fluctuation and therefore larger coherence loss. However, scatter is very large, more than an order of magnitude of coherence time at a certain PWV, suggesting that phase fluctuation is not a simple function of the amount of water vapor in the atmosphere. This result is the same as Sect. 3.2.3, since we are showing similar data with different expressions.

The trend appears to depend on frequency bands; Band 3 has larger scatter with less dependence on PWV, but Bands 6 and 7 have smaller scatter with tight dependence on PWV (see the fitted lines in Fig. 6). This can be explained as follows; observations at Band 3 can be carried out under a wider range of PWV, but for Bands 6 and 7 , the observation conditions are limited to better weather and the phase fluctuation quickly gets worse as weather condition gets worse.

\section{SUMMARY}

Using quasar stare data taken at the ALMA long baseline campaigns in the past 5 years, which has baseline lengths of up to $15 \mathrm{~km}$, we derived various atmospheric phase characteristics that will be useful for the ALMA long baseline (and also for higher frequency) observations. The summary of our study is listed as follows:

- The $183 \mathrm{GHz}$ water vapor radiometer (WVR) phase correction method works well for reducing the phase fluctuation at long baselines, especially for weather condition with precipitable water vapor (PWV) larger than $1 \mathrm{~mm}$.

- The WVR phase correction lengthens the coherence time by about a factor of two or more, indicating that the WVR phase correction is useful for lengthening the integration time and/or calibration time.

- The WVR phase correction, however, could not take out all the phase fluctuation, suggesting that there are other reasons that cause phase fluctuation at millimeter and submillimeter waves. Combining other phase correction methods in addition to the WVR phase correction is important, such as the band-to-band and/or the fast switching phase correction methods.

- Most of the spatial strucuture functions (SSFs) of the data show one turn-over around the baseline length of $1 \mathrm{~km}$. This result suggests that the scale height of the turbulent constituent is around $1 \mathrm{~km}$, consistent with the distribution of water vapor. Combine with the success of the WVR phase correction, it is obvious that the main turbulent constituent is water vapor in the atmosphere.

- The fitted slopes indicate that most of the phase fluctuation at the baseline length shorter than $1 \mathrm{~km}$ shows the intermediate value of theoretical 3D and 2D Kolmogorov turbulence (i.e., around 0.6), and that at the baseline length longer than $1 \mathrm{~km}$ displays the intermediate value of theoretical 2D Kolmogorov turbulence and no correlation (i.e., $0.2-0.3$ ).

- There are a few cases that SSFs do not show any turn-over. This SSF only obtained under very low PWV, when the WVR phase correction does not work well, or after the WVR phase correction. This suggests that the turbulent constituent is not water vapor. The scale height of this constituent is higher than 10 $\mathrm{km}$, suggesting that water ice or a dry component $\left(\mathrm{N}_{2}\right.$ or $\left.\mathrm{O}_{2}\right)$ can be the cause of the phase fluctuation.

- Empirical relations between the root-mean-square (rms) excess path length and the baseline length has been derived for the baseline length shorter and longer than $1 \mathrm{~km}$, and with and without the WVR phase correction. This will allow us to roughly estimate the phase fluctuation at the ALMA site. 
- Excess path length fluctuation at the baseline length of $10 \mathrm{~km}$ is large, $\sim 200 \mu \mathrm{m}$ even at PWV less than $1 \mathrm{~mm}$ (no large difference for the data before and after the WVR phase correction). This value is significant for the high frequency observations, and strongly suggest to use other phase correction methods, as mentioned above.

\section{ACKNOWLEDGMENTS}

SM and YA thank Joint ALMA Observatory (JAO) for supporting our visit to ALMA as Expert Visitors in 2013 and 2014. YA also expresses his appreciation to the National Astronomical Observatory of Japan (NAOJ) for their financial support for his stay in Chile in 2014. SM is supported by the National Science Council (NSC) and the Ministry of Science and Technology (MoST) of Taiwan, NSC 100-2112-M-001-006-MY3 and MoST 103-2112-M-001-032-MY3.

\section{REFERENCES}

[1] Hills, R. E., Kurz, R. J., and Peck, A. B., "ALMA: status report on construction and early results from commissioning," in [Ground-based and Airborne Telescopes III], Proc. SPIE 7733, 773317 (July 2010).

[2] ALMA Partnership, Fomalont, E. B., Vlahakis, C., Corder, S., Remijan, A., Barkats, D., Lucas, R., Hunter, T. R., Brogan, C. L., Asaki, Y., and et al., "The 2014 ALMA Long Baseline Campaign: An Overview," Astrophys. J. Letters 808, L1 (July 2015).

[3] ALMA Partnership, Hunter, T. R., Kneissl, R., Moullet, A., Brogan, C. L., Fomalont, E. B., Vlahakis, C., Asaki, Y., Barkats, D., Dent, W. R. F., and et al., "The 2014 ALMA Long Baseline Campaign: Observations of Asteroid 3 Juno at 60 Kilometer Resolution," Astrophys. J. Letters 808, L2 (July 2015).

[4] AlMA Partnership, Brogan, C. L., Pérez, L. M., Hunter, T. R., Dent, W. R. F., Hales, A. S., Hills, R. E., Corder, S., Fomalont, E. B., Vlahakis, C., and et al., "The 2014 ALMA Long Baseline Campaign: First Results from High Angular Resolution Observations toward the HL Tau Region," Astrophys. J. Letters 808, L3 (July 2015).

[5] A LMA Partnership, Vlahakis, C., Hunter, T. R., Hodge, J. A., Pérez, L. M., Andreani, P., Brogan, C. L., Cox, P., Martin, S., Zwaan, M., and et al., "The 2014 ALMA Long Baseline Campaign: Observations of the Strongly Lensed Submillimeter Galaxy HATLAS J090311.6+003906 at z = 3.042," Astrophys. J. Letters 808, L4 (July 2015).

[6] Sramek, R. A., "Atmospheric phase stability at the VLA," in [URSI/IAU Symposium on Radio Astronomical Seeing], Baldwin, J. E. and Wang, S., eds., 21-30 (1990).

[7] Carilli, C. L. and Holdaway, M. A., "Tropospheric phase calibration in millimeter interferometry," Radio Science 34, 817-840 (1999).

[8] Wright, M. C. H., "Atmospheric Phase Noise and Aperture Synthesis Imaging at Millimeter Wavelengths," Publ. Astron. Soc. Pacific 108, 520 (June 1996).

[9] Asaki, Y., Shibata, K. M., Kawabe, R., Roh, D.-G., Saito, M., Morita, K.-I., and Sasao, T., "Phase compensation experiments with the paired antennas method 2. Millimeter-wave fringe correction using centimeter-wave reference," Radio Science 33, 1297-1318 (1998).

[10] Matsushita, S. and Chen, Y.-L., "Testing the Atacama Compact Array Phase-Correction Scheme Using the Submillimeter Array," Publ. Astron. Soc. Japan 62, 1053-1062 (Aug. 2010).

[11] Matsushita, S., Morita, K.-I., Barkats, D., Hills, R. E., Fomalont, E. B., and Nikolic, B., "ALMA temporal phase stability and the effectiveness of water vapor radiometer," in [Ground-based and Airborne Telescopes IV], Proc. SPIE 8444, 84443E (Sept. 2012).

[12] Matsushita, S., Asaki, Y., Kawabe, R., Fomalont, E., Barkats, D., and Corder, S., "Phase characteristics of the ALMA 3-km baseline data," in [Ground-based and Airborne Telescopes V], Proc. SPIE 9145, 91453I (July 2014).

[13] Asaki, Y., Matsushita, S., Morita, K.-I., and Nikolic, B., "ACA phase calibration scheme with the ALMA water vapor radiometers," in [Ground-based and Airborne Telescopes IV], Proc. SPIE 8444, 84443K (Sept. 2012). 
[14] Asaki, Y., Matsushita, S., Kawabe, R., Fomalont, E., Barkats, D., and Corder, S., "ALMA fast switching phase calibration on long baselines," in [Ground-based and Airborne Telescopes V], Proc. SPIE 9145, 91454K (July 2014).

[15] Wiedner, M. C., Hills, R. E., Carlstrom, J. E., and Lay, O. P., "Interferometric Phase Correction Using 183 GHz Water Vapor Monitors," Astrophys. J. 553, 1036-1041 (June 2001).

[16] Nikolic, B., Bolton, R. C., Graves, S. F., Hills, R. E., and Richer, J. S., "Phase correction for ALMA with $183 \mathrm{GHz}$ water vapour radiometers," Astron. Astrophys. 552, A104 (Apr. 2013).

[17] Taylor, G. I., "The Spectrum of Turbulence," Proceedings of the Royal Society of London Series A 164, 476-490 (Feb. 1938).

[18] Dravskikh, A. F. and Finkelstein, A. M., "Tropospheric limitations in phase and frequency coordinate measurements in astronomy," Astrophys. Space Sci. 60, 251-265 (Feb. 1979).

[19] Asaki, Y., Saito, M., Kawabe, R., Morita, K.-I., and Sasao, T., "Phase compensation experiments with the paired antennas method," Radio Science 31, 1615-1626 (1996).

[20] McMullin, J. P., Waters, B., Schiebel, D., Young, W., and Golap, K., "CASA Architecture and Applications," in [Astronomical Data Analysis Software and Systems XVI], Shaw, R. A., Hill, F., and Bell, D. J., eds., Astronomical Society of the Pacific Conference Series 376, 127 (Oct. 2007).

[21] Nikolic, B., Graves, S. F., Bolton, R. C., and Richer, J. S., "Design and Implementation of the wvrgcal Program," ALMA Memo 593, ALMA (2012).

[22] Sramek, R. and Haupt, C., "ALMA System Technical Requirements for 12m array," Tech. Rep. ALMA80.04.00.00-005-B-SPE, ALMA (2006).

[23] Tatarskii, V. I., [Wave Propagation in Turbulent Medium], McGraw-Hill (1961).

[24] Thompson, A. R., Moran, J. M., and Swenson, Jr., G. W., [Interferometry and Synthesis in Radio Astronomy, 2nd Edition], Wiley-Interscience (2001).

[25] Butler, B. J., Radford, S. J. E., Sakamoto, S., and Kohno, K., "Atmospheric Phase Stability At Chajnantor and Pampa la Bola," ALMA Memo 365, ALMA (2001).

[26] Kameno, S., Nakai, N., and Honma, M., "ALMA Extended Array," in [New Trends in Radio Astronomy in the ALMA Era: The 30th Anniversary of Nobeyama Radio Observatory], Kawabe, R., Kuno, N., and Yamamoto, S., eds., Astronomical Society of the Pacific Conference Series 476, 409 (Oct. 2013).

[27] Morita, K.-I., Handa, K., Asaki, Y., Kitamura, Y., Yokogawa, S., Saito, M., Wilner, D. W., and Ho, P. T. P., "Fast Switching Experiments with the Nobeyama Millimeter Array," in [Imaging at Radio through Submillimeter Wavelengths], Mangum, J. G. and Radford, S. J. E., eds., Astronomical Society of the Pacific Conference Series 217, 340 (2000).

[28] Asaki, Y., Matsushita, S., Fomalont, E., Corder, S. A., Nyman, L.-A., Dent, W. R. F., Philips, N. M., Hirota, A., Takahashi, S., Vila-Vilaro, B., Nikolic, B., Hunter, T. R., Remijan, A., and Vlahakis, C., "ALMA long baseline phase calibration using phase referencing," in [Ground-based and Airborne Telescopes VI], Proc. SPIE 9906, in press (Paper 9906-224) (2016).

[29] Ray, P. S., "Broadband complex refractive indices of ice and water," App. Opt. 11, 1836 (Aug. 1972).

[30] Liebe, H. J., "MPM - An atmospheric millimeter-wave propagation model," International Journal of Infrared and Millimeter Waves 10, 631-650 (June 1989).

[31] Liebe, H. J., Hufford, G. A., and Manabe, T., "A model for the complex permittivity of water at frequencies below 1 THz," International Journal of Infrared and Millimeter Waves 12, 659-675 (July 1991).

[32] Matsushita, S., Matsuo, H., Sakamoto, A., and Pardo, J. R., "FTS measurements of submillimeter opacity and other site testing at Pampa la Bola," in [Radio Telescopes], Butcher, H. R., ed., Proc. SPIE 4015, 378-389 (July 2000).

[33] Matsushita, S. and Matsuo, H., "FTS Measurements of Submillimeter-Wave Atmospheric Opacity at Pampa la Bola: III. Water Vapor, Liquid Water, and $183 \mathrm{GHz}$ Water Vapor Line Opacities," Publ. Astron. Soc. Japan 55, 325-333 (Feb. 2003).

[34] Hufford, G., "A model for the complex permittivity of ice at frequencies below $1 \mathrm{THz}$," International Journal of Infrared and Millimeter Waves 12, 677-682 (July 1991).

[35] Liebe, H. J., Hufford, G. A., and Cotton, M. G., eds., [Propagation modeling of moist air and suspended water/ice particles at frequencies below $1000 \mathrm{GHz}$ (Nov. 1993). 